\title{
Generalized Gambler's Ruin Problem: Explicit Formulas via Siegmund Duality
}

\author{
Paweł Lorek ${ }^{1}$ iD
}

Received: 8 October 2015 / Revised: 20 February 2016

Accepted: 17 June 2016 / Published online: 8 July 2016

(C) The Author(s) 2016. This article is published with open access at Springerlink.com

\begin{abstract}
We give explicit formulas for ruin probabilities in a multidimensional Generalized Gambler's ruin problem. The generalization is best interpreted as a game of one player against $d$ other players, allowing arbitrary winning and losing probabilities (including ties) depending on the current fortune with particular player. It includes many previous other generalizations as special cases. Instead of usually utilized first-step-like analysis we involve dualities between Markov chains. We give general procedure for solving ruin-like problems utilizing Siegmund duality in Markov chains for partially ordered state spaces studied recently in context of Möbius monotonicity.
\end{abstract}

Keywords Generalized gambler's ruin problem · Markov chains ·

Absorption probability $\cdot$ Siegmund duality $\cdot$ Möbius monotonicity $\cdot$ Partial ordering

Mathematics Subject Classification (2010) $60 \mathrm{~J} 10 \cdot 60 \mathrm{G} 40 \cdot 60 \mathrm{~J} 80$

\section{Introduction}

Gambler's ruin problem has been playing important role in applied mathematics. There are applications in some casino games, e.g. craps (Isaac 1995), blackjack (Snell 2009), physics (El-Shehawey 2000; Yamamoto 2013), hydrology (Tsai et al. 2014), biology and epidemic models (Harik et al. 1999), finance (Scott 1981; Rolski et al. 2009; Asmussen

Work supported by NCN Research Grant DEC-2013/10/E/ST1/00359

Paweł Lorek

Pawel.Lorek@math.uni.wroc.pl

1 Mathematical Institute, University of Wrocław, pl. Grunwaldzki 2/4, 50-384, Wrocław, Poland 
and Albrecher 2010), just to mention few. There are many variations of the problem, newer ones are formulated after older ones are solved. For example, the following variations were proposed: infinite amount of money, three or more players (Kmet and Petkovšek 2002; Rocha and Stern 2004), the attrition variation (applies, e.g., to World Series or Stanley Cup finals Kaigh 1979), some cases of winning probabilities being dependent on the current fortune (El-Shehawey 2009; Lefebvre 2008).

The problem is as relevant today as it was in 17th century. According to (Edwards 1983) Pascal was the first who posed a problem in 1656 in a letter to Fermat. The most common form comes from Huygens who restated the problem as follows (rephrased):

"Let two men play with three dice, the first player scoring a point whenever 11 is thrown, whereas the second whenever 14 is thrown. Each player starts with 12 points. Successful roll adds one point to the player and subtracts one from the other player. The loser of the game is the first to reach zero points. What is the probability of victory for each player?"

Huygens gave a solution of above problem. (Bernoulli 1713) generalized and replaced Huygens' numerical results by formulas, i.e., he considered general initial capital $i$, general total amount of money $N$ and general winning probabilities $p \in(0,1)$. Since then the problem became very popular and different proofs were obtained. Pascal gave his solution to Cercavi without mentioning the method. According to Edwards (1983) Fermat probably used the combinatorial argument (as in "Problem of points"), most methods which appeared later used some kind of first-step-like analysis which led to solving some recursions.

It is known that the absorption probability of given chain can be related to the stationary distribution of some other ergodic chain. The relation is given via so-called Siegmund duality, the notion introduced in (Siegmund 1976). It was studied in financial context, where the probability that a dual risk process starting at level $h$ is ruined equals the probability that the stationary queue length exceeds level $h$, see (Asmussen and Albrecher 2010; Asmussen and Sigman 2009). Already in (Lindley 1952) such duality between some random walks on integers was shown. For this duality reader is also referred to (Theodore Cox and Rösler 1984; Diaconis and Fill 1990; Dette et al. 1997) or (Huillet 2010), just to mention few. All above papers have one thing in common: they study Siegmund duality defined for linear ordering of the state space (and most of them birth and death chains only). In this case (Siegmund 1976) states that the process has such dual if and only if it is stochastically monotone (w.r.t. total ordering). It is a little bit surprising that it was not exploited in the context of onedimensional Gambler's ruin problem. The solutions of the classical problem and its various one-dimensional generalizations are special cases of Theorem 1 and can be relatively easy calculated using usual stochastic monotonicity. On the other hand multidimensional case is quite different. For partial nonlinear ordering the stochastic monotonicity does not imply the existence of Siegmund dual, see (Liggett 2004). Finding such duals was successful for some specific chains and/or orderings. For example, in financial context, in (Błaszczyszyn and Sigman 1999) authors considered $R^{d}$-valued Markov processes (their Siegmund dual was set-valued). Recently, (Huillet 2014) considered dualities for Markov chains on partitions and sets. In (Lorek 2016) we show that Siegmund dual exists if and only if chain is Möbius monotone, the connections with Strong Stationary Duality (consult Diaconis and Fill 1990) are also given therein. Let us mention at this point that for non-linear ordering Möbius and stochastic monotonicities are, in general, different. In particular, we can have a chain which is not stochastically monotone, but which is Möbius monotone, thus we are able to construct its Siegmund dual. 
In this paper, based on results from (Lorek 2016), we give the solution to the multidimensional Generalized Gambler's ruin problem. The paper is organized as follows. In Section 2 we describe our Generalized Gambler's ruin problem, state its solution (Theorem 1) and point out other results as some special cases. In Section 3 we recall notion of Siegmund duality and antiduality for chains on partially ordered state spaces and give a general recipe for calculating ruin-like probabilities (summarized in Theorem 2). Section 4 includes some toy example (Cat Eats Mouse Eats Cheese), where the case of negative antidual matrix is presented. Finally, Section 5 contains proof of Theorem 1.

\section{Generalized Gambler's Ruin Problem and main Result}

In the one-dimensional Gambler's ruin problem two players start a game with total amount of, say, $N$ dollars and initial values $k$ and $N-k$. At each step they flip the coin (not necessary unbiased) to decide who wins a dollar. The game is over when one of them goes bankrupt.

We will consider the following generalization. There is one player (referred as "we") playing with $d \geq 1$ other players. Our initial assets are $\left(i_{1}, \ldots, i_{d}\right)$ with $0 \leq i_{j} \leq N_{j}, j=$ $1, \ldots, d\left(N_{j} \geq 1\right.$ is a total amount of assets with player $\left.j\right)$ and assets of consecutive players are $\left(N_{1}-i_{1}, \ldots, N_{d}-i_{d}\right)$. Then, with probability $p_{j}\left(i_{j}\right)$ we win one dollar with player $j$ and with probability $q_{j}\left(i_{j}\right)$ we lose it. With the remaining probability $1-\sum_{k=1}^{d}\left(p_{k}\left(i_{k}\right)+q_{j}\left(i_{k}\right)\right)$ we do nothing (i.e., ties are also possible). Once we win completely with player $j$ (i.e., $i_{j}=N_{j}$ ) we do not play with him/her anymore. We lose the whole game if we lose with at least one player, i.e., when $i_{j}=0$ for some $j=1, \ldots, d$. Note that if initially for some $j$ we have $i_{j}=0$ then we already lost (the winning probability is 0 ), thus we can restrict initial assets to $1 \leq i_{j} \leq N_{j}, j=1, \ldots, d$.

For $d=1$ and $p_{1}(j)=p, q_{1}(j)=q, N_{1}=N$ we have the classical Gambler's ruin problem on the state space $\{0, \ldots, N\}$. Many one-dimensional generalizations of this game were considered, e.g., (Lefebvre 2008) studied the case of some specific sequences of $p_{1}(j), q_{1}(j)$, later this was extended to any $p_{1}(j), q_{1}(j)$ in (El-Shehawey 2009). Variations of classical problem with ties allowed, i.e., $p_{1}(j)=p_{1}, q_{1}(j)=q_{1}, p_{1}+q_{1}<1$ were considered in, e.g., (Lengyel 2009a; 2009b) (the latter one considers so called conditional version of the problem). Some of the articles studied both, the ruin probability and duration of the game, whereas most papers studied only duration of the game. Some generalizations to a higher a dimension $d \geq 1$ were studied in (Rocha and Stern 2004; Kmet and Petkovšek 2002).

We will describe the game more formally as a Markov chain $Z^{\prime}$ with two absorbing states. The state space is $\mathbb{E}^{\prime}=\left\{\left(i_{1}, \ldots, i_{d}\right): 1 \leq i_{j} \leq N_{j}, 1 \leq j \leq d\right\} \cup\{-\infty\}$ (where $-\infty$ means we lose). For convenience denote $p_{j}\left(N_{j}\right)=q_{j}\left(N_{j}\right)=0=p_{j}(0)=q_{j}(0), j=1, \ldots, d$. Assume that for all $i_{j} \in\left\{1, \ldots, N_{j}-1\right\}, j=1, \ldots, d$ we have $p_{j}\left(i_{j}\right)>0, q_{j}\left(i_{j}\right)>0$ and $\sum_{k=1}^{d}\left(p_{k}\left(i_{k}\right)+q_{k}\left(i_{k}\right)\right) \leq 1$. With some abuse of notation, we will sometimes write $\left(i_{1}^{\prime}, \ldots, i_{d}^{\prime}\right)=-\infty$. The transitions of the described chain are following:

$\mathbf{P}_{Z^{\prime}}\left(\left(i_{1}, \ldots, i_{d}\right),\left(i_{1}^{\prime}, \ldots, i_{d}^{\prime}\right)\right)= \begin{cases}p_{j}\left(i_{j}\right) & \text { if } i_{j}^{\prime}=i_{j}+1, i_{k}^{\prime}=i_{k}, k \neq j, \\ q_{j}\left(i_{j}\right) & \text { if } i_{j}^{\prime}=i_{j}-1, i_{k}^{\prime}=i_{k}, k \neq j, \\ \sum_{j: i_{j}=1} q_{j}(1) & \text { if }\left(i_{1}^{\prime}, \ldots, i_{d}^{\prime}\right)=-\infty, \\ 1-\sum_{k=1}^{d}\left(p_{k}\left(i_{k}\right)+q_{k}\left(i_{k}\right)\right) & \text { if } i_{j}^{\prime}=i_{j}, 1 \leq j \leq d, \\ 1 & \text { if }\left(i_{1}, \ldots, i_{d}\right)=\left(i_{1}^{\prime}, \ldots, i_{d}^{\prime}\right)=-\infty .\end{cases}$ 
The chain, as required, has two absorbing states: $\left(N_{1}, \ldots, N_{d}\right)$ (we win) and $-\infty$ (we lose). We will give formulas for the probabilities of winning starting at arbitrary state, i.e., for

$$
\rho\left(\left(i_{1}, \ldots, i_{d}\right)\right)=P\left(\tau_{\left(N_{1}, \ldots, N_{d}\right)}<\tau_{-\infty} \mid Z_{0}^{\prime}=\left(i_{1}, \ldots, i_{d}\right)\right),
$$

where $\tau_{\mathbf{e}}:=\inf \left\{n \geq 0: Z_{n}^{\prime}=\mathbf{e}\right\}$. Our main result is following.

Theorem 1 Consider the Generalized Gambler's ruin problem described above. Then, the probability of winning starting at $\left(i_{1}, \ldots, i_{d}\right)$ is given by

$$
\rho\left(\left(i_{1}, \ldots, i_{d}\right)\right)=\frac{\prod_{j=1}^{d}\left(\sum_{n_{j}=1}^{i_{j}} \prod_{r=1}^{n_{j}-1}\left(\frac{q_{j}(r)}{p_{j}(r)}\right)\right)}{\prod_{j=1}^{d}\left(\sum_{n_{j}=1}^{N_{j}} \prod_{r=1}^{n_{j}-1}\left(\frac{q_{j}(r)}{p_{j}(r)}\right)\right)} .
$$

Theorem 1 generalizes some previous one-dimensional cases. For example:

- (i) Assume we have won with all the players except player $j$. Then, the probability of winning is

$$
\rho\left(\left(N_{1}, \ldots N_{j-1}, i_{j}, N_{j+1}, \ldots, N_{d}\right)\right)=\frac{\sum_{n=1}^{i_{j}} \prod_{r=1}^{n-1}\left(\frac{q_{j}(r)}{p_{j}(r)}\right)}{\sum_{n=1}^{N_{j}} \prod_{r=1}^{n-1}\left(\frac{q_{j}(r)}{p_{j}(r)}\right)} .
$$

This way formula (4.1) from (El-Shehawey 2009) is recovered (with some slight modification in notation, since authors considered a various versions of reflecting barriers).

- (ii) In addition to (i), let $p_{j}(r)=p, q_{j}(r)=q, r=1, \ldots, N_{j}$. Then we recover winning probability in the the classical Gambler's ruin problem (with possible ties):

$$
\rho\left(\left(N_{1}, \ldots N_{j-1}, i_{j}, N_{j+1}, \ldots, N_{d}\right)\right)=\frac{\sum_{n=1}^{i_{j}}\left(\frac{q}{p}\right)^{n-1}}{\sum_{n=1}^{N_{j}}\left(\frac{q}{p}\right)^{n-1}}= \begin{cases}\frac{1-\left(\frac{q}{p}\right)^{i_{j}}}{1-\left(\frac{q}{p}\right)^{N_{j}}} \text { if } p \neq q, \\ \frac{i_{j}}{N_{j}} \quad \text { otherwise. }\end{cases}
$$

- (iii) (Homogeneity case). Assume that for all $j=1, \ldots, d$ we have $p_{j}(r)=$ $p_{j}, q_{j}(r)=q_{j}, r=1, \ldots, N_{j}$. Define

$$
\mathcal{S}_{j}:=\left\{\begin{array}{l}
1 \text { if } p_{j}=q_{j}, \\
0 \text { otherwise }
\end{array}\right.
$$

Then we have

$$
\rho\left(\left(i_{1}, \ldots, i_{d}\right)\right)=\prod_{j=1}^{d}\left\{\left(\frac{1-\left(\frac{q_{j}}{p_{j}}\right)^{i_{j}}}{1-\left(\frac{q_{j}}{p_{j}}\right)^{N_{j}}}\right) \mathcal{S}_{j}+\frac{i_{j}}{N_{j}}\left(1-\mathcal{S}_{j}\right)\right\},
$$


which is a multidimensional generalization of the classical Gambler's ruin problem. Of course we obtain the same probabilities if only ratios $\frac{q_{j}\left(i_{j}\right)}{p_{j}\left(i_{j}\right)}$ are constant, e.g., for the following spatially nonhomogeneous case

$$
p_{j}\left(i_{j}\right)=\frac{p_{j}}{2 \rho i_{j}+1}, \quad q_{j}\left(i_{j}\right)=\frac{q_{j}}{2 \rho i_{j}+1},
$$

which is thus a multidimensional generalization of cases considered in (El-Shehawey 2009 ) and in (Lefebvre 2008). In the latter article only symmetric case corresponding to $p_{j}=q_{j}=1 / 2$ was considered.

\section{Tools: Siegmund Duality and Antiduality}

We shortly recall notion of Siegmund duality, its applications to studying absorption probabilities and result concerning existence of Siegmund dual from (Lorek 2016). Let $X$ be a discrete-time Markov chain with transition matrix $\mathbf{P}_{X}$ and finite state space $\mathbb{E}=$ $\left\{\mathbf{e}_{1}, \ldots, \mathbf{e}_{M}\right\}$ partially ordered by $\preceq$ with unique minimal element $\mathbf{e}_{1}$ and unique maximal element $\mathbf{e}_{M}$. Assume it is ergodic with the stationary distribution $\pi$. For $A \subseteq \mathbb{E}$ define $\mathbf{P}_{X}(\mathbf{e}, A):=\sum_{\mathbf{e}^{\prime} \in A} \mathbf{P}_{X}\left(\mathbf{e}, \mathbf{e}^{\prime}\right)$ and similarly $\pi(A):=\sum_{\mathbf{e} \in A} \pi(\mathbf{e})$. Define also $\{\mathbf{e}\}^{\uparrow}:=\left\{\mathbf{e}^{\prime} \in \mathbb{E}: \mathbf{e} \preceq \mathbf{e}^{\prime}\right\},\{\mathbf{e}\}^{\downarrow}:=\left\{\mathbf{e}^{\prime} \in \mathbb{E}: \mathbf{e}^{\prime} \preceq \mathbf{e}\right\}$ and $\delta\left(\mathbf{e}, \mathbf{e}^{\prime}\right)=\mathbf{1}\left(\mathbf{e}, \mathbf{e}^{\prime}\right)$. We say that Markov chain $Z$ with transition matrix $\mathbf{P}_{Z}$ is the Siegmund dual of $X$ if

$$
\forall\left(\mathbf{e}_{i}, \mathbf{e}_{j} \in \mathbb{E}\right) \forall(n \geq 0) \quad \mathbf{P}_{X}^{n}\left(\mathbf{e}_{i},\left\{\mathbf{e}_{j}\right\}^{\downarrow}\right)=\mathbf{P}_{Z}^{n}\left(\mathbf{e}_{j},\left\{\mathbf{e}_{i}\right\}^{\uparrow}\right) .
$$

Note that we can find a matrix fulfilling (3) which is substochastic, since we may have for some $\mathbf{e}_{j}$ that $\sum_{\mathbf{e}_{i}} \mathbf{P}_{Z}\left(\mathbf{e}_{j}, \mathbf{e}_{i}\right)<1$. In a similar way as Siegmund (1976) did (he considered linear ordering only), we add then one extra absorbing state, say $-\infty$ (called a coffin state). Denote the resulting matrix by $\mathbf{P}_{Z^{\prime}}$ and define $\mathbf{P}_{Z^{\prime}}\left(\mathbf{e}_{j},-\infty\right)=1-\sum_{\mathbf{e}_{i}} \mathbf{P}_{Z^{\prime}}\left(\mathbf{e}_{j}, \mathbf{e}_{i}\right)$, $\mathbf{P}_{Z}\left(-\infty, \mathbf{e}_{j}\right)=\delta\left(-\infty, \mathbf{e}_{j}\right)$ and $\mathbf{P}_{Z^{\prime}}\left(\mathbf{e}, \mathbf{e}_{2}\right)=\mathbf{P}_{Z}\left(\mathbf{e}, \mathbf{e}_{2}\right)$ otherwise. Note that Eq. 3 implies that $\mathbf{e}_{M}$ is an absorbing state, thus $Z^{\prime}$ has two absorbing states. Taking limits as $n \rightarrow \infty$ on both sides of Eq. 3 we have

$$
\pi\left(\left\{\mathbf{e}_{j}\right\}^{\downarrow}\right)=\lim _{n \rightarrow \infty} \mathbf{P}_{Z^{\prime}}^{n}\left(\mathbf{e}_{j},\left\{\mathbf{e}_{i}\right\}^{\uparrow}\right)=P\left(\tau_{\mathbf{e}_{M}}<\tau_{\mathbf{e}_{-\infty}} \mid Z_{0}^{\prime}=\mathbf{e}_{j}\right),
$$

where $\tau_{\mathbf{e}}=\inf \left\{n: Z_{n}^{\prime}=\mathbf{e}\right\}$. This way the stationary distribution of ergodic chain is related to the absorption probabilities of its Siegmund dual.

For partial ordering $\preceq$ define matrix $\mathbf{C}\left(\mathbf{e}, \mathbf{e}^{\prime}\right)=\mathbf{1}\left(\mathbf{e} \preceq \mathbf{e}^{\prime}\right)$. Such matrix is always invertible, and its inverse $\mathbf{C}^{-1}$ is often denoted by $\mu$ (what we use throughout the paper) and called the Möbius function of ordering $\preceq$. Note that Eq. 3 for $n=1$ can be written as

$$
\mathbf{P}_{X} \mathbf{C}=\mathbf{C} \mathbf{P}_{Z}^{T} \text {. }
$$

The main result of (Lorek 2016) is that for given partial ordering $\preceq$ the Siegmund dual chain exists if and only if $X$ is Möbius monotone (see also Lorek and Szekli 2012 for more details on this monotonicity). In such a case, the Siegmund dual on $\mathbb{E}^{\prime}=\mathbb{E} \cup\{-\infty\}$ has transitions outside coffin state given by

$$
\mathbf{P}_{Z^{\prime}}=\left(\mathbf{C}^{-1} \mathbf{P}_{X} \mathbf{C}\right)^{T}
$$

(the nonnegativity of which is the definition of Möbius monotonicity of $X$ ). The natural application is in studying stationary distribution of a chain $X$ (e.g., its asymptotics): calculate Siegmund dual and then its probability of being eventually absorbed in $\mathbf{e}_{M}$. However, we can reverse the process starting with a chain $Z^{\prime}$ with two absorbing states (we win or we 
lose). Assume its state space is $\mathbb{E}^{\prime}=\{-\infty\} \cup\left\{\mathbf{e}_{1}, \ldots, \mathbf{e}_{M}\right\}$ with absorbing states $-\infty$ and $\mathbf{e}_{M}$. Denote $\mathbb{E}:=\left\{\mathbf{e}_{1}, \ldots, \mathbf{e}_{M}\right\}$.

The procedure is then the following:

1) remove state $-\infty$ obtaining substochastic matrix $\mathbf{P}_{Z}$;

2) introduce some partial ordering $\preceq$ expressed by matrix $\mathbf{C}$ such that $\mathbf{e}_{M}$ is a unique maximal element;

3) calculate transitions of Siegmund antidual chain $X$ from Eq. 5 calculating $\mathbf{P}_{X}=$ $\mathbf{C} \mathbf{P}_{Z}^{T} \mathbf{C}^{-1}$;

4) if the resulting matrix $\mathbf{P}_{X}$ has a stationary measure $\pi$ such that $\forall(\mathbf{e} \in \mathbb{E})$ $\lim _{n \rightarrow \infty} \mathbf{P}_{X}^{n}(\mathbf{e}, \cdot)=\pi(\cdot)$ then we can calculate absorption probabilities of $Z^{\prime}$ from the relation (4) (if $\mathbf{P}_{X}$ is a stochastic matrix, then $\pi$ is the stationary distribution of the chain related to this matrix).

The details are in the following theorem.

Theorem 2 Let $Z^{\prime}$ be a Markov chain with transition matrix $\mathbf{P}_{Z^{\prime}}$ on $\mathbb{E}^{\prime}=\{-\infty\} \cup$ $\left\{e_{1}, \ldots, \mathbf{e}_{M}\right\}=:\{-\infty\} \cup \mathbb{E}$ with two absorbing states $-\infty$ and $\mathbf{e}_{M}$. Consider substochastic kernel $\mathbf{P}_{Z}$ which is the matrix $\mathbf{P}_{Z^{\prime}}$ with row and column corresponding to $-\infty$ removed. Fix some partial ordering $\preceq$ on $\mathbb{E}$ expressed by matrix $\mathbf{C}$ such that $\mathbf{e}_{M}$ is a unique maximal state. Calculate

$$
\mathbf{P}_{X}=\mathbf{C} \mathbf{P}_{Z}^{T} \mathbf{C}^{-1} \text {. }
$$

The resulting $\mathbf{P}_{X}$ has the property that $\forall(\mathbf{e} \in \mathbb{E}) \sum_{\mathbf{e}_{2} \in \mathbb{E}} \mathbf{P}_{X}\left(\mathbf{e}, \mathbf{e}_{2}\right)=1$. Assume that there exists invariant measure $\pi$ fulfilling:

$$
\forall\left(\mathbf{e}_{2} \in \mathbb{E}\right) \lim _{n \rightarrow \infty} \mathbf{P}_{X}^{n}\left(\mathbf{e}_{2}, \mathbf{e}\right)=\pi(\mathbf{e}), \quad \sum_{\mathbf{e} \in \mathbb{E}} \pi(\mathbf{e})=1 .
$$

Then we have $\rho\left(\mathbf{e}^{\prime}\right):=P\left(\tau_{\mathbf{e}_{M}}<\tau_{-\infty} \mid Z_{0}^{\prime}=\mathbf{e}^{\prime}\right)=\pi\left(\left\{\mathbf{e}^{\prime}\right\}^{\downarrow}\right)$ (i.e., relation (4) holds).

Remark 1 If resulting $\mathbf{P}_{X}$ in Eq. 7 is a stochastic matrix of ergodic chain, say $X$, then $\pi$ is its stationary distribution. Moreover, it is Möbius monotone with respect to $\preceq$.

Remark 2 If the resulting $\mathbf{P}_{X}$ has negative entries it does not have a real probabilistic interpretation. However, e.g., in area of quantum mechanics, such "distributions", called negative quasi-probabilities are quite common and natural in this context. This notion was already introduced in (Wigner 1932), where author writes:

"[...] cannot be really interpreted as the simultaneous probability for coordinates and momenta, as is clear from the fact, that it may take negative values. But of course this must not hinder the use of it in calculations as an auxiliary function which obeys many relations we would expect from such a probability."

For some recent connections of negative quasi-probability and quantum computations see (Veitch et al. 2012).

Proof of Theorem 2 The main sketch of the proof was essentially given before the theorem. The only thing which may be not clear is that for all $\mathbf{e}$ we have $\sum_{\mathbf{e}^{\prime}} \mathbf{P}_{X}\left(\mathbf{e}, \mathbf{e}^{\prime}\right)=1$. Let us calculate

$$
\sum_{\mathbf{e}^{\prime}} \mathbf{P}_{X}\left(\mathbf{e}, \mathbf{e}^{\prime}\right)=\sum_{\mathbf{e}^{\prime}}\left(\mathbf{C P}_{Z}^{T} \mathbf{C}^{-1}\right)\left(\mathbf{e}, \mathbf{e}^{\prime}\right)=\sum_{\mathbf{e}^{\prime}} \sum_{\mathbf{e}_{2}}\left(\mathbf{C P}_{Z}^{T}\right)\left(\mathbf{e}, \mathbf{e}_{2}\right) \mathbf{C}^{-1}\left(\mathbf{e}_{2}, \mathbf{e}^{\prime}\right) .
$$


We have

$$
\begin{aligned}
\left(\mathbf{C} \mathbf{P}_{Z}^{T}\right)\left(\mathbf{e}, \mathbf{e}_{2}\right) & =\sum_{\mathbf{e}_{3}} \mathbf{C}\left(\mathbf{e}, \mathbf{e}_{3}\right) \mathbf{P}_{Z}^{T}\left(\mathbf{e}_{3}, \mathbf{e}_{2}\right)=\sum_{\mathbf{e}_{3}} \mathbf{C}\left(\mathbf{e}, \mathbf{e}_{3}\right) \mathbf{P}_{Z}\left(\mathbf{e}_{2}, \mathbf{e}_{3}\right) \\
& =\sum_{\mathbf{e}_{3} \succeq \mathbf{e}} \mathbf{P}_{Z}\left(\mathbf{e}_{2}, \mathbf{e}_{3}\right)=\mathbf{P}_{Z}\left(\mathbf{e}_{2},\left\{\mathbf{e}^{\uparrow}\right),\right.
\end{aligned}
$$

thus

$$
\begin{aligned}
\sum_{\mathbf{e}^{\prime}} \mathbf{P}_{X}\left(\mathbf{e}, \mathbf{e}^{\prime}\right) & =\sum_{\mathbf{e}^{\prime}} \sum_{\mathbf{e}_{2}} \mathbf{P}_{Z}\left(\mathbf{e}_{2},\{\mathbf{e}\}^{\uparrow}\right) \mu\left(\mathbf{e}_{2}, \mathbf{e}^{\prime}\right)=\sum_{\mathbf{e}^{\prime}} \mu\left(\mathbf{e}_{2}, \mathbf{e}^{\prime}\right) \sum_{\mathbf{e}_{2}} \mathbf{P}_{Z}\left(\mathbf{e}_{2},\{\mathbf{e}\}^{\uparrow}\right) \\
& \stackrel{(*)}{=} \mathbf{P}_{Z}\left(\mathbf{e}_{M},\{\mathbf{e}\}^{\uparrow}\right)=1 .
\end{aligned}
$$

In (*) we used the fact, that for any partial order with unique maximal element $\mathbf{e}_{M}$, the Möbius function fulfills $\forall(\mathbf{e} \in \mathbb{E}) \sum_{\mathbf{e}_{j}} \mathbf{C}^{-1}\left(\mathbf{e}, \mathbf{e}_{j}\right)=\mathbf{1}\left(\mathbf{e}=\mathbf{e}_{M}\right)$. To see this consider column of $\mathbf{C}^{-1}$ corresponding to state $\mathbf{e}_{M}$ after applying first elementary column operation of Gauss-Jordan elimination.

\section{Toy example: Cat Eats Mouse Eats Cheese}

Before proceeding to the proof of the main result on Generalized Gambler's ruin problem (i.e., Theorem 1) we give a 5-state example. The reason for this is that we wanted to present an example having the resulting matrix $\mathbf{P}_{X}$ with negative entries. The example is taken from (Bremaud 1999) (Example 3.2 Cat Eats Mouse Eats Cheese, where the answer is easily calculated using first-step analysis):

"A merry mouse moves in a maze. If it is at time $n$ in a room with $k$ adjacent rooms, it will be at time $n+1$ in one of the $k$ adjacent rooms, choosing one at random, each with probability $\frac{1}{k}$. A fat lazy cat remains all the time in a given room, and a piece of cheese waits for the mouse in another room (see Fig. 1). The cat is not completely

\begin{tabular}{|c|c|c|}
\hline 2 & 3 & \\
\hline 1 & 4 & $\begin{array}{l}5 \\
\text { CHEESE }\end{array}$ \\
\hline
\end{tabular}
lazy: If the mouse enters the room inhabited by the cat, the cat will eat it. What is the probability that the mouse ever gets to eat the cheese when starting from room 1 , the cat and the cheese being in rooms 3 and 5, respectively?"

Fig. 1 Maze, mouse, and murder 
Essentially we have $\mathbb{E}^{\prime}=\{3\} \cup\{1,2,4,5\}$ and

$\mathbf{P}_{Z^{\prime}}=\left(\begin{array}{ccccc}0 & \frac{1}{2} & 0 & \frac{1}{2} & 0 \\ \frac{1}{2} & 0 & \frac{1}{2} & 0 & 0 \\ 0 & 0 & 1 & 0 & 0 \\ \frac{1}{3} & 0 & \frac{1}{3} & 0 & \frac{1}{3} \\ 0 & 0 & 0 & 0 & 1\end{array}\right), \mathbf{P}_{Z}=\left(\begin{array}{cccc}0 & \frac{1}{2} & \frac{1}{2} & 0 \\ \frac{1}{2} & 0 & 0 & 0 \\ \frac{1}{3} & 0 & 0 & \frac{1}{3} \\ 0 & 0 & 0 & 1\end{array}\right), \mathbf{C}=\left(\begin{array}{llll}1 & 1 & 1 & 1 \\ 0 & 1 & 0 & 1 \\ 0 & 0 & 1 & 1 \\ 0 & 0 & 0 & 1\end{array}\right)={ }_{1}^{4}$,

where $\mathbf{P}_{Z^{\prime}}$ is the original Cat Eats Mouse Eats Cheese matrix, $\mathbf{P}_{Z}$ is the matrix with state 3 removed $(\mathbb{E}=\{1,2,4,5\})$ and $\mathbf{C}$ represents the ordering with Hasse diagram presented on the right side (with 5 being a maximal state). We are to calculate

$$
\rho(j)=P\left(\tau_{5}<\tau_{3} \mid Z_{0}^{\prime}=j\right), j=1,2,4,5 .
$$

Calculating $\mathbf{P}_{X}$ from Eq. 7 and its stationary measure gives

$$
\mathbf{P}_{X}=\mathbf{C P}_{Z}^{T} \mathbf{C}^{-1}=\left(\begin{array}{rrrr}
1 & -\frac{1}{2} & -\frac{1}{3} & \frac{5}{6} \\
\frac{1}{2} & -\frac{1}{2} & -\frac{1}{6} & \frac{7}{6} \\
\frac{1}{2} & -\frac{1}{2} & -\frac{1}{6} & \frac{7}{6} \\
0 & 0 & \frac{1}{3} & \frac{2}{3}
\end{array}\right),(\pi(1), \pi(2), \pi(4), \pi(5))=\left(\frac{2}{7},-\frac{1}{7}, \frac{1}{7}, \frac{5}{7}\right) .
$$

From Eq. 4 we have $(\rho(1), \rho(2), \rho(4), \rho(5))=\left(\frac{2}{7}, \frac{1}{7}, \frac{3}{7}, 1\right)$.

\section{Proof of Theorem 1}

From matrix $\mathbf{P}_{Z^{\prime}}$ given in Eq. 1 we remove state $-\infty$ obtaining the following substochastic matrix on $\mathbb{E}=\left\{1,2, \ldots, N_{1}\right\} \times \cdots \times\left\{1,2, \ldots, N_{d}\right\}$

$\mathbf{P}_{Z}\left(\left(i_{1}, \ldots, i_{d}\right),\left(i_{1}^{\prime}, \ldots, i_{d}^{\prime}\right)\right)= \begin{cases}p_{j}\left(i_{j}\right) & \text { if } i_{j}^{\prime}=i_{j}+1, i_{k}^{\prime}=i_{k}, k \neq j, \\ q_{j}\left(i_{j}\right) & \text { if } i_{j}^{\prime}=i_{j}-1, i_{k}^{\prime}=i_{k}, k \neq j, \\ 1-\sum_{k=1}^{d}\left(p_{k}\left(i_{k}\right)+q_{k}\left(i_{k}\right)\right) & \text { if } i_{j}^{\prime}=i_{j}, 1 \leq j \leq d .\end{cases}$

Consider the coordinate-wise ordering: $\left(i_{1}, \ldots, i_{d}\right) \preceq\left(i_{1}^{\prime}, \ldots, i_{d}^{\prime}\right)$ iff $i_{j} \leq i_{j}^{\prime}, j=$ $1, \ldots, d$. The state $\mathbf{e}_{M}:=\left(N_{1}, \ldots, N_{d}\right)$ is a unique maximal one. Directly from Proposition 5 in (Rota 1964) we find the corresponding Möbius function

$\mu\left(\left(i_{1}, \ldots, i_{d}\right),\left(i_{1}+r_{1}, \ldots, i_{d}+r_{d}\right)\right)= \begin{cases}(-1)^{\sum_{k=1}^{n} r_{k}} & r_{j} \in\{0,1\}, i_{j}+r_{j} \leq N_{j}, j=1, \ldots, d \\ 0 & \text { otherwise. }\end{cases}$

We have: $\mathbf{P}_{X}\left(\left(i_{1}, \ldots, i_{d}\right),\left(i_{1}^{\prime}, \ldots, i_{d}^{\prime}\right)\right)=\mathbf{C} \mathbf{P}_{Z}^{T} \mathbf{C}^{-1}\left(\left(i_{1}, \ldots, i_{d}\right),\left(i_{1}^{\prime}, \ldots, i_{d}^{\prime}\right)\right)$

$$
=\sum_{\left(i_{1}^{(2)}, \ldots, i_{d}^{(2)}\right) \leq\left(i_{1}^{\prime}, \ldots, i_{d}^{\prime}\right)} \mu\left(\left(i_{1}^{(2)}, \ldots, i_{d}^{(2)}\right),\left(i_{1}^{\prime}, \ldots, i_{d}^{\prime}\right)\right) \mathbf{P}_{Z}\left(\left(i_{1}^{(2)}, \ldots, i_{d}^{(2)}\right),\left\{\left(i_{1}, \ldots, i_{d}\right)\right\}^{\uparrow}\right) .
$$

Let $\mathbf{s}_{j}=(0, \ldots, 0,1,0, \ldots, 0)$, where 1 is on position $j$. Consider the following cases: 
- $\quad$ Case $\left(i_{1}^{\prime}, \ldots, i_{d}^{\prime}\right)=\left(i_{1}, \ldots, i_{d}\right)-\mathbf{s}_{j}$. Then we have $\mathbf{P}_{X}\left(\left(i_{1}, \ldots, i_{d}\right),\left(i_{1}^{\prime}, \ldots, i_{d}^{\prime}\right)\right)=$

$$
\begin{aligned}
= & \sum_{\substack{\left(i_{1}^{(2)}, \ldots, i_{d}^{(2)}\right) \leq\left(i_{1}, \ldots, i_{d}\right)-\mathbf{s}_{j} \\
=}} \mu\left(\left(i_{1}^{(2)}, \ldots, i_{d}^{(2)}\right),\left(i_{1}, \ldots, i_{d}\right)-\mathbf{s}_{j}\right) \mathbf{P}_{Z}\left(\left(i_{1}^{(2)}, \ldots, i_{d}^{(2)}\right),\left\{\left(i_{1}, \ldots, i_{d}\right)\right\}^{\uparrow}\right) \\
& \mu\left(\left(i_{1}, \ldots, i_{d}\right)-\mathbf{s}_{j},\left(i_{1}, \ldots, i_{d}\right)-\mathbf{s}_{j}\right) \mathbf{P}_{Z}\left(\left(i_{1}, \ldots, i_{d}\right)-\mathbf{s}_{j},\left\{\left(i_{1}, \ldots, i_{d}\right)\right\}^{\uparrow}\right) \\
& +\sum_{\substack{\left(i_{1}^{(2)}, \ldots, i_{d}^{(2)}\right) \leq\left(i_{1}, \ldots, i_{d}\right)-\mathbf{s}_{j} \\
\left(i_{1}^{(2)}, \ldots, i_{d}^{(2)}\right) \neq\left(i_{1}, \ldots, i_{d}\right)-\mathbf{s}_{j}}} \mu\left(\left(i_{1}^{(2)}, \ldots, i_{d}^{(2)}\right),\left(i_{1}, \ldots, i_{d}\right)-\mathbf{s}_{j}\right) \mathbf{P}_{Z}\left(\left(i_{1}^{(2)}, \ldots, i_{d}^{(2)}\right),\left\{\left(i_{1}, \ldots, i_{d}\right)\right\}^{\uparrow}\right) . \\
= & 1 \cdot \mathbf{P}_{Z}\left(\left(i_{1}, \ldots, i_{d}\right)-\mathbf{s}_{j},\left(i_{1}, \ldots, i_{d}\right)\right)+0=q_{j}\left(i_{j}-1\right) .
\end{aligned}
$$

- $\quad$ Case $\left(i_{1}^{\prime}, \ldots, i_{d}^{\prime}\right)=\left(i_{1}, \ldots, i_{d}\right)+\mathbf{s}_{j}$. We have $\mathbf{P}_{X}\left(\left(i_{1}, \ldots, i_{d}\right),\left(i_{1}^{\prime}, \ldots, i_{d}^{\prime}\right)\right)=$

$$
\begin{aligned}
& =\sum_{\left(i_{1}^{(2)}, \ldots, i_{d}^{(2)}\right) \leq\left(i_{1}, \ldots, i_{d}\right)+\mathbf{s}_{j}} \mu\left(\left(i_{1}^{(2)}, \ldots, i_{d}^{(2)}\right),\left(i_{1}, \ldots, i_{d}\right)+\mathbf{s}_{j}\right) \mathbf{P}_{Z}\left(\left(i_{1}^{(2)}, \ldots, i_{d}^{(2)}\right),\left\{\left(i_{1}, \ldots, i_{d}\right)\right\}^{\uparrow}\right) \\
& =\mu\left(\left(i_{1}, \ldots, i_{d}\right)+\mathbf{s}_{j},\left(i_{1}, \ldots, i_{d}\right)+\mathbf{s}_{j}\right) \mathbf{P}_{Z}\left(\left(i_{1}, \ldots, i_{d}\right)+\mathbf{s}_{j},\left\{\left(i_{1}, \ldots, i_{d}\right)\right\}^{\uparrow}\right) \\
& +\mu\left(\left(i_{1}, \ldots, i_{d}\right),\left(i_{1}, \ldots, i_{d}\right)+\mathbf{s}_{j}\right) \mathbf{P}_{Z}\left(\left(i_{1}, \ldots, i_{d}\right),\left\{\left(i_{1}, \ldots, i_{d}\right)\right\}^{\uparrow}\right) \\
& +\sum_{\substack{r=1, \ldots, d \\
r \neq j}} \mu\left(\left(i_{1}, \ldots, i_{d}\right)-\mathbf{s}_{r}+\mathbf{s}_{j},\left(i_{1}, \ldots, i_{d}\right)+\mathbf{s}_{j}\right) \mathbf{P}_{Z}\left(\left(i_{1}, \ldots, i_{d}\right)-\mathbf{s}_{r}+\mathbf{s}_{j},\left\{\left(i_{1}, \ldots, i_{d}\right)\right\}^{\uparrow}\right) \\
& +\sum_{\substack{r=1, \ldots, d \\
r \neq j}} \mu\left(\left(i_{1}, \ldots, i_{d}\right)-\mathbf{s}_{r},\left(i_{1}, \ldots, i_{d}\right)+\mathbf{s}_{j}\right) \mathbf{P}_{Z}\left(\left(i_{1}, \ldots, i_{d}\right)-\mathbf{s}_{r},\left\{\left(i_{1}, \ldots, i_{d}\right)\right\}^{\uparrow}\right) \\
& =(-1)^{0} \cdot \mathbf{P}_{Z}\left(\left(i_{1}, \ldots, i_{d}\right)+\mathbf{s}_{j},\left\{\left(i_{1}, \ldots, i_{d}\right)\right\}^{\uparrow}\right)+(-1)^{1} \cdot \mathbf{P}_{Z}\left(\left(i_{1}, \ldots, i_{d}\right),\left\{\left(i_{1}, \ldots, i_{d}\right)\right\}^{\uparrow}\right) \\
& +\sum_{\substack{r=1, \ldots, d \\
r \neq j}}\left[(-1)^{1} \cdot \mathbf{P}_{Z}\left(\left(i_{1}, \ldots, i_{d}\right)-\mathbf{s}_{r}+\mathbf{s}_{j},\left\{\left(i_{1}, \ldots, i_{d}\right)\right\}^{\uparrow}\right)\right. \\
& \left.+(-1)^{2} \cdot \mathbf{P}_{Z}\left(\left(i_{1}, \ldots, i_{d}\right)-\mathbf{s}_{r},\left\{\left(i_{1}, \ldots, i_{d}\right)\right\}^{\uparrow}\right)\right] \\
& =1-\sum_{\substack{r=1, \ldots, d \\
r \neq j}} q_{r}\left(i_{r}\right)-\left(1-\sum_{r=1} q_{r}\left(i_{r}\right)\right)+\sum_{\substack{r=1, \ldots, d \\
r \neq j}}(-1+1) p_{r}\left(i_{r}\right)=q_{j}\left(i_{j}\right) .
\end{aligned}
$$

- $\quad$ Case $\left(i_{1}^{\prime}, \ldots, i_{d}^{\prime}\right)=\left(i_{1}, \ldots, i_{d}\right)$. We have $\mathbf{P}_{X}\left(\left(i_{1}, \ldots, i_{d}\right),\left(i_{1}, \ldots, i_{d}\right)\right)=$

$$
\begin{aligned}
& =\quad \sum_{\left(i_{1}^{(2)}, \ldots, i_{d}^{(2)}\right) \leq\left(i_{1}, \ldots, i_{d}\right)} \mu\left(\left(i_{1}^{(2)}, \ldots, i_{d}^{(2)}\right),\left(i_{1}, \ldots, i_{d}\right)\right) \mathbf{P}_{Z}\left(\left(i_{1}^{(2)}, \ldots, i_{d}^{(2)}\right),\left\{\left(i_{1}, \ldots, i_{d}\right)\right\}^{\uparrow}\right) \\
& =\mu\left(\left(i_{1}, \ldots, i_{d}\right),\left(i_{1}, \ldots, i_{d}\right)\right) \mathbf{P}_{Z}\left(\left(i_{1}, \ldots, i_{d}\right),\left\{\left(i_{1}, \ldots, i_{d}\right)\right\}^{\uparrow}\right) \\
& +\sum_{r=1, \ldots, d} \mu\left(\left(i_{1}, \ldots, i_{d}\right)-\mathbf{s}_{r},\left(i_{1}, \ldots, i_{d}\right)\right) \mathbf{P}_{Z}\left(\left(i_{1}, \ldots, i_{d}\right),\left\{\left(i_{1}, \ldots, i_{d}\right)\right\}^{\uparrow}\right) \\
& =(-1)^{0} \cdot \mathbf{P}_{Z}\left(\left(i_{1}, \ldots, i_{d}\right),\left\{\left(i_{1}, \ldots, i_{d}\right)\right\}^{\uparrow}\right)+\sum_{r=1, \ldots, d}(-1)^{1} \cdot \mathbf{P}_{Z}\left(\left(i_{1}, \ldots, i_{d}\right),\left\{\left(i_{1}, \ldots, i_{d}\right)\right\}^{\uparrow}\right) \\
& =1-\sum_{r=1, \ldots, d} q_{r}\left(i_{r}\right)-\sum_{r=1, \ldots, d} p_{r}\left(i_{r}-1\right)=1-\sum_{r=1, \ldots, d}\left(p_{r}\left(i_{r}-1\right)+q_{r}\left(i_{r}\right)\right) .
\end{aligned}
$$


These were the only nonzero entries of $\mathbf{P}_{X}$. Summarizing we have

$$
\mathbf{P}_{X}\left(\left(i_{1}, \ldots, i_{d}\right),\left(i_{1}^{\prime}, \ldots, i_{d}^{\prime}\right)\right)= \begin{cases}q_{j}\left(i_{j}\right) & \text { if } i_{j}^{\prime}=i_{j}+1, i_{k}^{\prime}=i_{k}, k \neq j, \\ p_{j}\left(i_{j}-1\right) & \text { if } i_{j}^{\prime}=i_{j}-1, i_{k}^{\prime}=i_{k}, k \neq j, \\ 1-\sum_{k=1}^{d}\left(p_{k}\left(i_{k}-1\right)+q_{k}\left(i_{k}\right)\right) & \text { if } i_{j}^{\prime}=i_{j}, 1 \leq j \leq d .\end{cases}
$$

Assume that for all $\left(i_{1}, \ldots, i_{d}\right)$ we have $\mathbf{P}_{X}\left(\left(i_{1}, \ldots, i_{d}\right),\left(i_{1}, \ldots, i_{d}\right)\right) \geq 0$. Then, these are the transitions of a closed network with $d$ independent servers: being at state $\left(i_{1}, \ldots, i_{d}\right)$ the arrival to server $j$ is $q_{j}\left(i_{j}\right)$ and the departure is $p_{j}\left(i_{j}-1\right)$. Its stationary distribution is following

$$
\pi\left(\left(i_{1}, \ldots, i_{d}\right)\right)=\frac{\prod_{j=1}^{d}\left(\prod_{r=1}^{i_{j}-1}\left(\frac{q_{j}(r)}{p_{j}(r)}\right)\right)}{\prod_{j=1}^{d}\left(\sum_{n_{j}=1}^{N_{j}} \prod_{r=1}^{n_{j}-1}\left(\frac{q_{j}(r)}{p_{j}(r)}\right)\right)} .
$$

However, if some entry of $\mathbf{P}_{X}$ is negative, then of course the matrix does not represent any Markov chain, but with $\pi$ given in Eq. 8 we still have that $\pi \mathbf{P}_{X}=\pi$ and $\lim _{n \rightarrow \infty} \mathbf{P}_{X}^{n}\left(\left(i_{1}, \ldots, i_{d}\right), \cdot\right)=\pi(\cdot)$ for every $\left(i_{1}, \ldots, i_{d}\right) \in \mathbb{E}$.

Thus, because of Theorem 2 equality (4) holds in any case and we obtain (2) what finishes the proof.

Acknowledgments Author thanks Zbigniew Palmowski for helpful suggestions and discussions. Partial support by the project RARE-318984, a Marie Curie IRSES Fellowship within the 7th European Community Framework Programme, is kindly acknowledged.

Open Access This article is distributed under the terms of the Creative Commons Attribution 4.0 International License (http://creativecommons.org/licenses/by/4.0/), which permits unrestricted use, distribution, and reproduction in any medium, provided you give appropriate credit to the original author(s) and the source, provide a link to the Creative Commons license, and indicate if changes were made.

\section{References}

Asmussen S, Albrecher H (2010) Ruin Probabilities

Asmussen S, Sigman K (2009) Monotone Stochastic Recursions and their Duals. Probab Eng Inf Sci 10(01):1 Bernoulli J (1713) Ars conjectandi

Błaszczyszyn B, Sigman K (1999) Risk and duality in multidimensions. Stochastic processes and their applications 83:331-356

Bremaud P (1999) Markov chains: Gibbs fields, Monte Carlo simulation, and queues

Diaconis P, Fill JA (1990) Strong stationary times via a new form of duality. Ann Probab 18(4):1483-1522

Dette H, Fill JA, Pitman J, Studden WJ (1997) Wall and siegmund duality relations for birth and death chains with reflecting barrier. J Theor Probab 10(2):349-374

Edwards AW (1983) Pascal's problem: The 'Gambler's ruin'. Int Stat Rev 51:73-79

El-Shehawey MA (2009) On the gambler's ruin problem for a finite Markov chain. Statistics \& Probability Letters 79(14):1590-1595

El-Shehawey MA (2000) Absorption probabilities for a random walk between two partially absorbing boundaries: I. J Phys A Math Gen 33(49):9005-9013

Harik G, Cantú-Paz E, Goldberg DE, Miller BL (1999) The gambler's ruin problem, genetic algorithms, and the sizing of populations. Evol Comput 7(3):231-253

Huillet T (2010) Siegmund duality with applications to the neutral Moran model conditioned on never being absorbed. J Phys A Math Theor 43:37 
Huillet T (2014) Servet Martínez On Möbius Duality and Coarse-Graining. Journal of Theoretical Probability Isaac R (1995) The pleasures of probability. Undergraduate texts in mathematics. Springer new york, new york, NY

Kaigh WD (1979) An attrition problem of gambler's ruin. Math Mag 52:22-25

Kmet A, Petkovšek M (2002) Gambler's ruin problem in several dimensions. Adv Appl Math 28(2):107-118

Lefebvre M (2008) The gambler's ruin problem for a Markov chain related to the Bessel process. Statistics \& Probability Letters 78(15):2314-2320

Lengyel T (2009a) Gambler's ruin and winning a series by m games. Ann Inst Stat Math 63(1):181-195

Lengyel T (2009b) The conditional gambler's ruin problem with ties allowed. Appl Math Lett 22(3):351-355

Liggett TM (2004) Interacting particle systems

Lindley DV (1952) The theory of queues with a single server. Math Proc Camb Philos Soc 48(02):277-289

Lorek P (2016) Siegmund duality for Markov chains on partially ordered state spaces. Submitted:1-14

Lorek P, Szekli R (2012) Strong stationary duality for Möbius monotone Markov chains. Queueing Systems 71(1-2):79-95

Rocha AL, Stern F (2004) The asymmetric n-player gambler's ruin problem with equal initial fortunes. Adv Appl Math 33(3):512-530

Rolski T, Schmidli H, Schmidt V, Teugels J (2009) stochastic processes for insurance and finance

Rota G-C (1964) On the foundations of combinatorial theory I. Theory of Möbius functions. Probab Theory Relat Fields 368:340-368

Scott J (1981) The probability of bankruptcy. J Bank Financ 5(3):317-344

Siegmund D (1976) The equivalence of absorbing and reflecting barrier problems for stochastically monotone Markov processes The Annals of Probability

Snell JL (2009) Gambling, probability and martingales. The Mathematical Intelligencer 4(3):118-124

Theodore Cox J., Rösler U (1984) A duality relation for entrance and exit laws for Markov processes. Stochastic processes and their applications 16:141-156

Tsai CW, Hsu Y, Lai K-C, Wu N-K (2014) Application of gambler's ruin model to sediment transport problems. J Hydrol 510:197-207

Veitch V, Ferrie C, Gross D, Emerson J (2012) Negative quasi-probability as a resource for quantum computation. New J Phys 14(11):113011

Wigner E (1932) On the quantum correction for thermodynamic equilibrium. Phys Rev 40(5):749-759

Yamamoto K (2013) Solution and analysis of a One-Dimensional First-Passage problem with a nonzero halting probability. International Journal of Statistical Mechanics 2013:1-9 